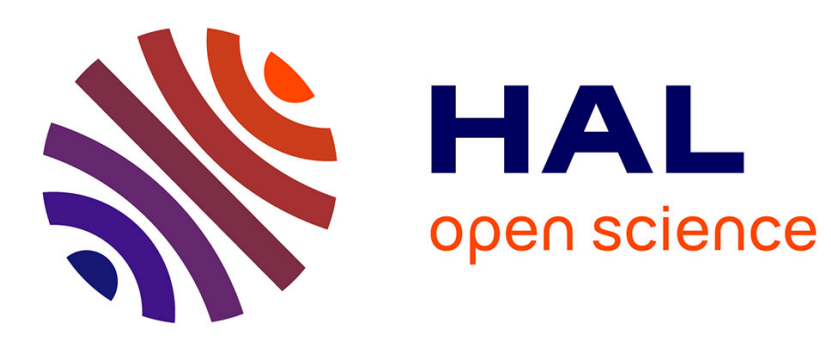

\title{
Fusion of multi-tracer PET images for dose painting
}

Benoit Lelandais, Su Ruan, Thierry Denoeux, Pierre Vera, Isabelle Gardin

\section{To cite this version:}

Benoît Lelandais, Su Ruan, Thierry Denoeux, Pierre Vera, Isabelle Gardin. Fusion of multitracer PET images for dose painting. Medical Image Analysis, 2014, 18 (7), pp.1247-1259. 10.1016/j.media.2014.06.014 . hal-01127787

\section{HAL Id: hal-01127787 \\ https://hal.science/hal-01127787}

Submitted on 8 Mar 2015

HAL is a multi-disciplinary open access archive for the deposit and dissemination of scientific research documents, whether they are published or not. The documents may come from teaching and research institutions in France or abroad, or from public or private research centers.
L'archive ouverte pluridisciplinaire HAL, est destinée au dépôt et à la diffusion de documents scientifiques de niveau recherche, publiés ou non, émanant des établissements d'enseignement et de recherche français ou étrangers, des laboratoires publics ou privés. 


\title{
Fusion of multi-tracer PET images for Dose Painting
}

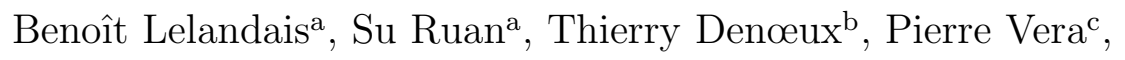 \\ Isabelle Gardin ${ }^{\mathrm{c}}$ \\ ${ }^{a}$ QuantIF, LITIS EA 4108, University of Rouen, France. \\ ${ }^{b}$ Heudiasyc (UMR 7253), Université de Technologie de Compiègne, CNRS, Compiègne, \\ France. \\ ${ }^{c}$ Department of Nuclear medicine, Henri becquerel center, France.
}

\begin{abstract}
PET imaging with FluoroDesoxyGlucose (FDG) tracer is clinically used for the definition of Biological Target Volumes (BTVs) for radiotherapy. Recently, new tracers, such as FLuoroThymidine (FLT) or FluoroMisonidazol (FMiso), have been proposed. They provide complementary information for the definition of BTVs. Our work is to fuse multi-tracer PET images to obtain a good BTV definition and to help the radiation oncologist in dose painting. Due to the noise and the partial volume effect leading, respectively, to the presence of uncertainty and imprecision in PET images, the segmentation and the fusion of PET images is difficult. In this paper, a framework based on Belief Function Theory (BFT) is proposed for the segmentation of BTV from multi-tracer PET images. The first step is based on an extension of the Evidential C-Means (ECM) algorithm, taking advantage of neighboring voxels for dealing with uncertainty and imprecision in each mono-tracer PET image. Then, imprecision and uncertainty are, respectively, reduced using prior knowledge related to defects in the acquisition system and neighborhood information. Finally, a multi-tracer PET image fusion is performed. The results are represented by a set of parametric maps that provide important information for dose painting. The performances are evaluated on PET phantoms and patient data with lung cancer. Quantitative results show good performance of our method compared with other methods.
\end{abstract}

Keywords: Dose Painting, Positron Emission Tomography, Information Fusion, Segmentation, Belief Function Theory. 


\section{Introduction}

Medical imaging plays an important role in radiotherapy for treatment planning with the delineation of Gross Tumor Volume (GTV) based on Computed Tomography images. A promising approach to increasing local control of the tumor without increasing normal tissue exposure is to take advantage of intra-tumor heterogeneity using functional imaging. Hall has proposed the concept of dose painting, which involves application of a dose prescription based on biological characteristics of the tumor (Hall, 2005). To this end, Positron Emission Tomography (PET) is used for sub-volumes definition, also called Biological Target Volumes (BTV) (Ling et al., 2000). In the last decade, technological innovations, such as the invention of Intensity Modulated Radiation Therapy (IMRT), made a highly precise radiation application possible (Thorwarth, 2012). As a consequence, high conformal radiation doses can now be given to the tumor while sparing organs at risk and normal tissues. IMRT is based on multileaf collimators leading to a radiation with a spatial resolution in the same order of magnitude than PET images (few millimetres $(5-7 \mathrm{~mm}))$. Dose painting is the subject of a lot of research on its technical feasibility (Alber et al., 2003), the potential benefit in term of dose escalation within the tumor (Thorwarth et al., 2007), respect for dose constraints to organs at risk (Choi et al., 2010), as clinical validation (Duprez et al., 2011).

The main radio-tracer proposed in the literature for dose painting is ${ }^{18}$ F-FluoroDesoxyGlucose (FDG), due to the enhanced glucose metabolism of tumor cells corresponding to a high uptake, also called FDG positive tissue. Several other new radio-tracers are of interest, such as ${ }^{18}$ F-FLuoroThymidine (FLT) to visualize areas corresponding to a high level of cell proliferation (Yang et al., 2010), and ${ }^{18}$ F-FluoroMisonidazol (FMiso) to visualize tumoral regions that have an inadequate supply of oxygen (hypoxic regions) and are radioresistant (Chang et al., 2009). Dose prescription could possibly be adapted in accordance with the radio-tracers. For example, an increase of dose frequency deliverance could be planned for high proliferative cells (i.e., FLT positive tissue), or the dose can be boosted for FMiso positive tissues. Thus, the definition of BTV in each tracer image and their fusion is important to make the radiotherapy more efficient.

Although many methods have been proposed for segmenting positive tissue on PET images in the literature, there is no consensus on this issue. 
Most of the methods are proposed to delineate only FDG-PET positive tissues. Example include thresholding methods (Erdi et al., 1997; Daisne et al., 2003; Black et al., 2004; Nestle et al., 2005; Vauclin et al., 2009) and region growing methods (Haiying et al., 2006; Krohn et al., 2007; Potesil et al., 2007; Green et al., 2008). The disadvantage of these methods is their sensitivity to noise and heterogeneous uptake of positive tissues. Watershed algorithms are also proposed in (Riddell et al., 1999; Tylski et al., 2006; Geets et al., 2007). However, they are sensitive to noise because they are based on gradient measure. Some methods based on probabilistic measures (Aristophanous et al., 2007; Hatt et al., 2009) and fuzzy measures (Zaidi et al., 2002; DewalleVignion et al., 2011) have recently been proposed. Their advantages are the capability of dealing with noise and/or partial volume effect which is due to the low spatial resolution of acquisition system and the post-filtering applied on PET images. However, in the face of the problems due to heterogeneous uptake of positive tissues, these methods fail. The segmentation of FLT and FMiso-PET images is more complicated than FDG-PET images (see Figure 1). In (Hatt et al., 2010), the authors propose to use their probabilistic method (Hatt et al., 2009) for the segmentation of FLT-PET images. For FMiso-PET positive tissue segmentation, a statistical approach based on artificial ants moving in a three dimensional image space has been proposed (Haase et al., 2012). This specific method has been proposed to address low contrast images. To date, there is no method reported to address more than three tracers. Such a method is challenging to develop due to the partial volume effect, the possibly high noise and the low contrast in PET images.

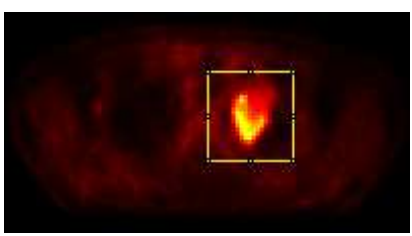

(a) FDG

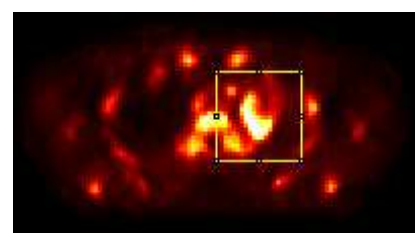

(b) FLT

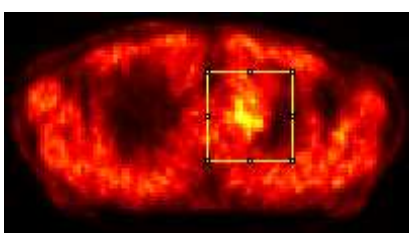

(c) FMiso

Figure 1: FDG, FLT and FMiso PET images for one patient.

In this paper, we provide a tool to fuse multi-tracer PET images for achieving a good dose painting.

As probability and possibility theories, Belief Function Theory (BFT) is a theoretical framework for reasoning with partial knowledge (Dempster, 1967; Shafer, 1976; Smets and Kennes, 1994). Moreover, it has the advantage to 
contain powerful combination operators (Smets, 1993; Denœux, 2008). In previous work (Lelandais et al., 2012), we have shown that BFT is a good tool to address multi PET tracers. Our method used BFT to address the imperfections by combining neighboring voxels in a Fuzzy C-Means (FCM) algorithm and to combine multi-tracer PET images. In this paper, we propose a new method that is also based on BFT. The belief masses relative to the tumor are estimated by an extension of the Evidential C-Means (ECM) algorithm which is widely used in data classification to address uncertainty and imprecision. Spatial information is added to the classical ECM to reduce uncertainty due to noise. A new objective function is proposed to design a new algorithm named Spatial Evidential C-Means (SECM) algorithm. Our method is divided into three steps: 1) modeling of uncertainty and imprecision in each mono-tracer PET-image, using spatial information. 2) Reduction of imprecision and uncertainty by combining a measure of partial volume effect specific to the acquisition system and neighborhood information. 3) Fusion of multi-tracer PET images to build parametric maps that describe the locations of glucose metabolism, cell proliferation and hypoxia of tumor.

The manuscript is organized as follow. The proposed framework is presented in the next section. A brief presentation of the BFT is also given in this section. The results on PET images are given in section 3 followed by a conclusion.

\section{Material and methods}

As our method is based on belief function theory, we first present it in the following section. Then, the method is presented.

\subsection{Belief Function Theory}

\subsubsection{Concept}

As probability and possibility theories, belief function theory is a theoretical framework for reasoning with partial and unreliable information. Let $\Omega=\left\{\omega_{1}, \omega_{2}, \ldots, \omega_{c}\right\}$ be a finite set of classes, called the frame of discernment. Partial knowledge obtained from information sources is taken into account by assigning Basic Belief Assignments (BBAs) or masses to different sub-

sets of the frame of discernment. Function $m$ is defined as a mapping from $2^{\Omega}=\left\{\varnothing,\left\{\omega_{1}\right\},\left\{\omega_{2}\right\}, \ldots,\left\{\omega_{c}\right\},\left\{\omega_{1}, \omega_{2}\right\}, \ldots, \Omega\right\}$ to $[0,1]$ verifying:

$$
\sum_{A \subseteq \Omega} m(A)=1 .
$$


A hypothesis $A$ is called a focal set if $m(A) \neq 0$. The mass $m(\Omega)$ represents the degree of ignorance $(m(\Omega)=1$ corresponds to a total ignorance) and the mass $m(\varnothing)$ is used for representing the conflict.

Using this framework, one can distinguish singletons, such as $\left\{\omega_{1}\right\}$, from disjunctions, such as $\left\{\omega_{1}, \omega_{2}\right\}$ corresponding to a set of exclusive classes. Singletons allow us to represent uncertainty as in probability theory. A BBA is considered as uncertain when masses are approximately equally distributed over singletons, corresponding to equiprobability in probability theory. Disjunctions allow us to represent imprecision. A BBA is considered as imprecise when non-empty masses are assigned over disjunctions, implying that we do not take part in favor of one class or another.

\subsubsection{Basic Belief Assignment (BBA) transformation}

From a BBA one can determine a belief function (bel) and a plausibility function $(p l)$ such as:

$$
\begin{aligned}
\text { bel: }: 2^{\Omega} & \longrightarrow[0,1] \\
A & \longmapsto \sum_{B \subseteq A, B \neq \varnothing} m(B), \\
p l: 2^{\Omega} & \longrightarrow[0,1] \\
A & \longmapsto \sum_{B \cap A \neq \varnothing} m(B) .
\end{aligned}
$$

These two functions can be used in the last stage of a process of decisionmaking. For decision making, one can also use the pignistic probability function $(B e t P)$ (Smets and Kennes, 1994) defined by:

$$
\begin{aligned}
\operatorname{Bet} P: 2^{\Omega} & \longrightarrow[0,1] \\
A & \longmapsto \sum_{B \subseteq \Omega} \frac{|A \cap B|}{|B|} \frac{m(B)}{1-m(\varnothing)} .
\end{aligned}
$$

where |.| denotes the cardinality of the considered set. The idea is to convert a

BBA into a probability function by redistributing the masses on disjunctions towards singletons.

\subsubsection{Combination of BBAs}

Two BBAs coming from two information sources and defined on the same frame of discernment $\Omega$ can be pooled using combination rules.

Let $S_{1}$ and $S_{2}$ be two reliable sources of information. The conjunctive combination rule is given by:

$$
m_{S_{1} \odot S_{2}}(A)=\sum_{B \cap C=A} m_{1}(B) \cdot m_{2}(C) .
$$


It allows us to reduce uncertainty and imprecision by transferring belief masses to conjunctions of hypotheses. When the information contained in sources are in conflict, masses are transferred on $\varnothing$ in the open world assumption. In order to satisfy $m(\varnothing)=0$ (closed world assumption (Smets and Kennes, 1994)), the Dempster's rule is used (Dempster, 1967):

$$
m_{S_{1} \oplus S_{2}}(A)=\frac{1}{1-\kappa} \sum_{B \cap C=A} m_{1}(B) \cdot m_{2}(C) .
$$

where $\kappa$ is the mass on the empty set in the open world assumption. Dempster's rule involves normalizing the conjunctive rule by the conflict between sources.

Both the conjunctive and Dempster's rules are commutative and associative, and can also be generalized to $N$ sources of information.

\subsection{Evidential C-Means (ECM)}

The ECM algorithm has been introduced in (Masson and Denœux, 2008) for dealing with partial knowledge in the context of BFT framework. Let $\left\{\mathbf{x}_{1}, \ldots, \mathbf{x}_{i}, \ldots, \mathbf{x}_{n}\right\}$ be a collection of vectors in $\mathbb{R}^{p}$ describing the $n$ objects. Let $c$ be the desired number of classes. Each cluster is represented by a prototype or a center $\mathbf{v}_{k} \in \mathbb{R}^{p}$ associated with each class $\omega_{k}$. Then, for each subset $A_{j} \subseteq \Omega, A_{j} \neq \varnothing$, a centroid $\overline{\mathbf{v}}_{j}$ is calculated as the barycenter of the prototypes associated with the classes in $A_{j}$ :

$$
\overline{\mathbf{v}}_{j}=\frac{1}{c_{j}} \sum_{k=1}^{c} s_{k j} \mathbf{v}_{k}
$$

with:

$$
s_{k j}= \begin{cases}1 & \text { if } \omega_{k} \in A_{j} \\ 0 & \text { otherwise }\end{cases}
$$

where $c_{j}=\left|A_{j}\right|$ denotes the cardinal of $A_{j}$. The distance $d_{i j}$ between $\mathbf{x}_{i}$ and the focal set $A_{j}$ is defined by:

$$
d_{i j}^{2}=\left\|\mathbf{x}_{i}-\overline{\mathbf{v}}_{j}\right\|^{2}
$$

Let $V$ denotes a matrix of size $(c \times p)$ composed of the coordinates of the cluster centers such that $V_{k q}$ is the $q$ th component of the cluster center $\mathbf{v}_{j}$. ECM looks for a partition matrix $M=\left(m_{i j}\right)\left(A_{j} \neq \varnothing, A_{j} \subseteq \Omega\right)$, called a credal 
partition, of size $\left(n \times 2^{c}\right)$ and for the matrix $\mathbf{V}$ by minimizing the following objective function:

$$
J_{E C M}(M, V)=\sum_{i=1}^{n} \sum_{\left\{j / A_{j} \neq \varnothing, A_{j} \subseteq \Omega\right\}} c_{j}^{\alpha} m_{i j}^{\beta} d_{i j}^{2}+\sum_{i=1}^{n} \delta^{2} m_{i \varnothing}^{\beta}
$$

subject to:

$$
\sum_{\left\{j / A_{j} \neq \varnothing, A_{j} \subseteq \Omega\right\}} m_{i j}+m_{i \varnothing}=1 \quad \forall i=1, n .
$$

where $m_{i \varnothing}$ denote $m_{i}(\varnothing)$, and where $\delta$ is a weighting parameter. The empty set is used for outliers. Coefficient $\alpha \geq 0$ controls the degree of penalization of the subsets according to their cardinality ( $\alpha=2$ by default) and coefficient $\beta>1$ controls the fuzziness of the partition ( $\beta=2$ by default).

\subsection{Proposed method: Spatial Evidential C-Means (SECM)}

For deriving a credal partition from image data, we propose adding spatial information to the ECM. Some methods (Ahmed et al., 2002; Chen and Zhang, 2004) have been proposed to derive a fuzzy partition in image processing by using neighborhood information. Because the performance of ECM has shown superiority in many cases, we propose to extend it by integrating spatial information. Let $\Phi(i)=\{1, \ldots, t, \ldots, T\}$ the set of the $T$ neighbors of a voxel $i$, including $i$. Let $t \in \Phi(i)$ be a neighbor of $i$ and let $b_{t}$ be a weight function that varies in $[0,1]$ according to the spatial distance between $t$ and $i$. $b_{t}$ controls the neighborhood influence and is defined as follow:

$$
b_{t}=\exp \left(\frac{-d_{i t}^{2}}{2 \cdot \eta^{2}}\right)
$$

where $\eta$ is a parameter controlling the influence of neighborhood. For our application, $\eta^{2}=\mathrm{FWHM}^{2} / 8 \log 2$, with FWHM (Full Width at Half Maximum) corresponding to the spatial resolution of PET images. $b_{t}$ equals 1 when $d_{i t}=0$, and decreases as $d_{i t}$ increases. For the distance $d_{i j}$ in equation

9 , we propose the weighted sum of the distances in $\Phi(i): \sum_{t \in \Phi(i)} b_{t} d_{t j}$. The objective function to be minimized is then given by:

$$
J_{S E C M}(M, V)=\sum_{i=1}^{n} \sum_{\left\{j / A_{j} \neq \varnothing, A_{j} \subseteq \Omega\right\}} c_{j}^{\alpha} m_{i j}^{\beta} \sum_{t \in \Phi(i)} b_{t} d_{t j}^{2}+\sum_{i=1}^{n} \delta^{2} m_{i \varnothing}^{\beta}
$$

subject to

$$
\sum_{\left\{j / A_{j} \neq \varnothing, A_{j} \subseteq \Omega\right\}} m_{i j}+m_{i \varnothing}=1 \quad \forall i=1, n,
$$


Note that $M$ and $V$ have been defined before. Using this objective function, the lower $\sum_{t \in \Phi(i)} b_{t} d_{t j}$ is, the higher the mass $m_{i j}=m_{i}\left(A_{j}\right)$ will be, and vice versa.

To model the imperfection from PET medical images, we suggest modifying the objective function slightly. We assume that all the voxels belong either to the background ( $\omega_{1}$ hypothesis) or to high uptake of radioactivity ( $\omega_{2}$ hypothesis). Thus, with the closed world assumption, $m(\varnothing)=0$, and the term corresponding to the outlier detection can be removed. The objective function becomes then:

$$
J_{S E C M}(M, V)=\sum_{i=1}^{n} \sum_{\left\{j / A_{j} \neq \varnothing, A_{j} \subseteq \Omega\right\}} c_{j}^{\alpha} m_{i j}^{\beta} \sum_{t \in \Phi(i)} b_{t} d_{t j}^{2}
$$

subject to:

$$
\sum_{\left\{j / A_{j} \neq \varnothing, A_{j} \subseteq \Omega\right\}} m_{i j}=1 \quad \forall i=1, n .
$$

To minimize $J_{S E C M}$, an alternate optimization scheme similar to those detailed in (Masson and Denœux, 2008) can be used. First, we consider that $V$ is fixed. We can show that $M$ can be updated using the following equation:

$$
m_{i j}=\frac{c_{j}^{-\alpha / \beta-1}\left(\sum_{t \in \Phi(i)} b_{t}^{1 /(\beta-1)} d_{t j}^{2 /(\beta-1)}\right)^{-1}}{\sum_{A_{k} \neq \varnothing} c_{k}^{-\alpha / \beta-1}\left(\sum_{t \in \Phi(i)} b_{t}^{1 /(\beta-1)} d_{t k}^{2 /(\beta-1)}\right)^{-1}}
$$

Then, we consider that $M$ is fixed. Let $B$ be a matrix of size $(c \times p)$ defined by:

$$
B_{l q}=\sum_{i} \sum_{t \in \Phi_{i}} b_{t} x_{t q} \sum_{A_{j} \ni \omega_{l}} c_{j}^{\alpha-1} m_{i j}^{\beta}, \quad l=1, c, q=1, p,
$$

and $H$ a matrix of size $(c \times c)$ given by:

$$
H_{l k}=\sum_{i} \sum_{A_{j} \supseteq\left\{\omega_{k}, \omega_{l}\right\}} c_{j}^{\alpha-2} m_{i j}^{\beta} \sum_{t \in \Phi_{i}} b_{t}, \quad k, l=1, c
$$

We can show that the minimization of $J_{S E C M}$ with respect to $V$ is an unconstrained optimization problem which can be simplified as:

$$
H V=B .
$$

This equation can be solved using a standard linear system solver. The proof is given in Appendix A. By alternatively computing $M$ using (17) and 


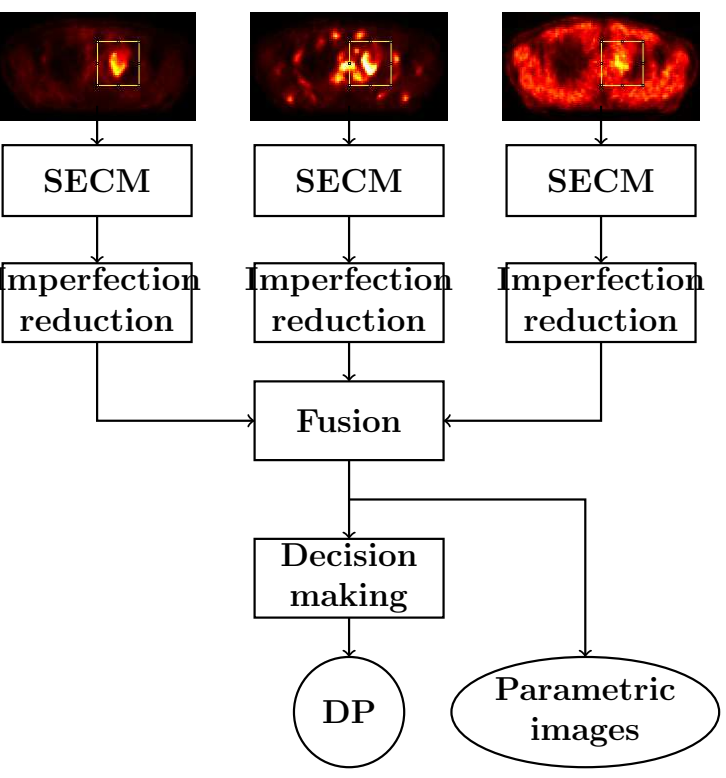

Figure 2: Poposed fusion sheme from multi-tracer PET images.

solving $V$ using (18), (19) and (20) in an iterative process, the convergence of the algorithm is guaranteed.

The process can be applied irrespective of the number of classes. It allows us to consider neighborhood information in deriving the credal partition from image data. For comparison, if ECM allows us to model both uncertainty and imprecision in a one-dimensional feature space (gray level), SECM models the imperfections in a $T$-dimensional feature space, where $T$ is the number of neighbors, in order to take into account spatial relationships.

\subsection{Proposed framework}

The proposed framework first processes each PET image using SECM to model both uncertainty and imprecision. Then, imprecision and uncertainty are reduced by a combination of different pieces of information. The fusion of the three PET images is finally performed to obtain a parametric map allowing us to help the radiation oncologist for dose painting purpose. The details of each step are given below.

\subsubsection{SECM for each PET image}

A tissue is said to be positive if it presents a high uptake with one tracer PET image. Two focal sets are considered: the back- 
ground and positive tissue, respectively identified by hypotheses $\left\{H_{b}\right\}$ and $\left\{H_{p}\right\}$. Because the belief function framework is used, a third focal set representing imprecision is also considered: $\left\{H_{b}, H_{p}\right\}$.

After applying SECM on PET images, basic belief masses are spread over hypotheses $\left\{H_{b}\right\},\left\{H_{p}\right\}$ and $\left\{H_{b}, H_{p}\right\}$. As a result, we obtain certain, uncertain and imprecise voxels, defined as follows: It results that:

- Certain voxels are those having a high belief mass in favor of $\left\{H_{b}\right\}$ or $\left\{H_{p}\right\}$.

- Uncertain voxels are those having a medium belief mass in favor of both $\left\{H_{b}\right\}$ and $\left\{H_{p}\right\}$.

- Imprecise voxels are those having a high belief mass in favor of $\left\{H_{b}, H_{p}\right\}$.

In PET images, the uncertainty comes from the noise, while the imprecision comes from two phenomena. The first one corresponds to partial volume effect that is present for voxels at the transition between two regions. The second one corresponds to the potential heterogeneity of positive tissues. Heterogeneity is reflected by the presence of inhomogeneity of gray levels in tumor region. Thus, the SECM algorithm allows us to separate these two problems: uncertainty due to noise and imprecision due to both partial volume effect and uptake heterogeneity.

\subsubsection{Imprecision reduction for each PET image}

As the partial volume effect is caused by the low spatial resolution of the acquisition system, we propose to measure this effect using PET phantom images. A Jaszczak phantom is used, which contains eight spheres whose volumes vary from 1.02 to $98.16 \mathrm{ml}$, and eight contrasts ranging from 1.7 to 22.9. Because the same acquisition system is used in both cases, PET phantom images and patient images have the same spatial resolution. Our objective is to reduce $\left\{H_{b}, H_{p}\right\}$ representing the imprecision. We define a coefficient $\gamma$ which reflects the part of imprecision due to the partial volume effect. This coefficient can be measured through the phantom because the sphere volumes are known a priori. The coefficient $\gamma$, corresponding to the part of belief from $\left\{H_{b}, H_{p}\right\}$ that should be transferred to $\left\{H_{p}\right\}$, is learned for each sphere according to its volume and its contrast with the background measured after applying SECM. The measurement is carried out by applying our SECM method on the phantom PET images, and by searching with 


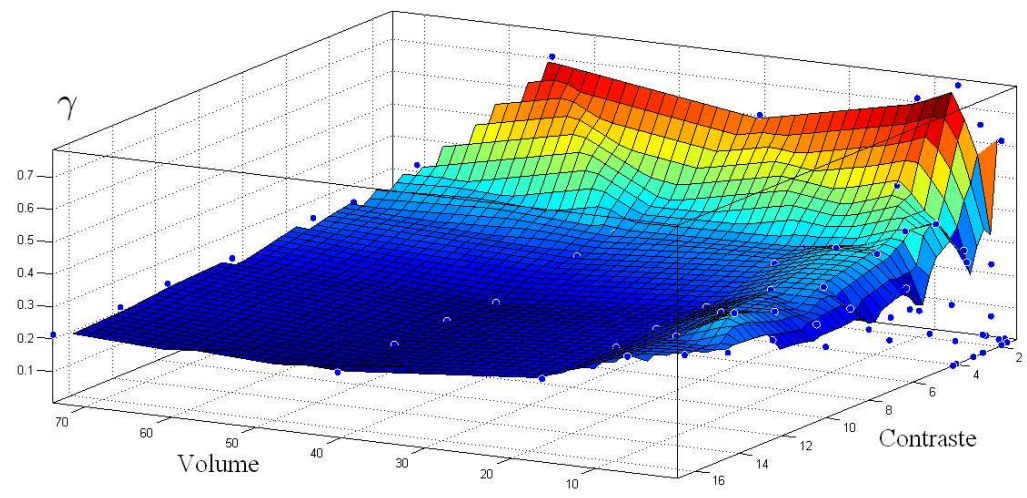

Figure 3: The value $\gamma$, learned according to the volume and the contrast of spheres.

a dichotomical process, the values allowing us to measure the true sphere volumes. The measurement result obtained is presented Figure 3. The lower both the contrast and the volume are, the higher $\gamma$ is, except for the smallest volumes.

The use of $\gamma$ for reducing imprecision is carried out by applying a reinforcement mechanism. First, we propose the following simple mass function having two focal elements for representing the a priori contextual knowledge:

$$
\begin{aligned}
m^{\gamma}\left(\left\{H_{p}\right\}\right) & =\gamma \\
m^{\gamma}\left(\left\{H_{b}, H_{p}\right\}\right) & =1-\gamma .
\end{aligned}
$$

where $H_{p}$ is the subset to reinforce. Then, the combination of each voxelsource with this BBA is applied using Dempster's rule:

$$
\mathrm{M}_{i}=m_{i} \oplus m^{\gamma}
$$

This process allows us to transfer a part of belief to the subset $\left\{H_{p}\right\}$ corresponding to the tumor in PET, and thus reduce imprecision. The reduction of imprecision is applied on the entire image. However, it has an influence for voxels where partial volume effect occurs (where $m\left(\left\{H_{b}, H_{p}\right\}\right)>>0$ ).

\subsubsection{Uncertainty reduction for each PET image}

Noise is important in PET images due to low contrast, a short acquisition time period and/or a low activity of the tracer. As proposed in (Capelle-Laize et al., 2004; Zhang et al., 2007; Makni et al., 2014; Lelandais et al., 2012), a solution for reducing noise in the belief function framework is to combine the neighborhood information. 
We propose to combine mass functions from neighboring voxels. First, a discounting is done according to the same coefficient $b_{t}$ as before, by transferring a part of belief on the set $\left\{H_{b}, H_{p}\right\}$ :

$$
\begin{aligned}
\mathscr{M}_{t}\left(\left\{H_{b}\right\}\right) & =b_{t} \mathrm{M}_{t}\left(H_{b}\right), \\
\mathscr{M}_{t}\left(\left\{H_{p}\right\}\right) & =b_{t} \mathrm{M}_{t}\left(H_{p}\right), \\
\mathscr{M}_{t}\left(\left\{H_{b}, H_{p}\right\}\right) & =1-b_{t}+b_{t} \mathrm{M}_{t}\left(\left\{H_{b}, H_{p}\right\}\right)
\end{aligned}
$$

The discounting operation allows us to reduce the influence of voxels that are physically far away from $i$ before the conjunctive combination. Then, in order to respect the closed world assumption, we propose to use Dempster's rule:

$$
\mathcal{M}_{i}(.)=\bigoplus_{t \in \Phi(i)} \mathscr{M}_{t}(.)
$$

The combination of neighbor voxels allows us to remove uncertainty due to noise, by transferring their belief on the singletons. Note that residual imprecision, after the previous step, is also reduced at this step.

\subsubsection{Decision for one PET image}

For segmenting one mono-tracer PET image, the plausibility function is computed (eq. 3) according to the two classes, and voxels are classified according to their highest plausibility value. Note that this process is not carried out if multi-tracer PET images are considered and fused. Algorithm 1 gives an overview of the proposed method. 


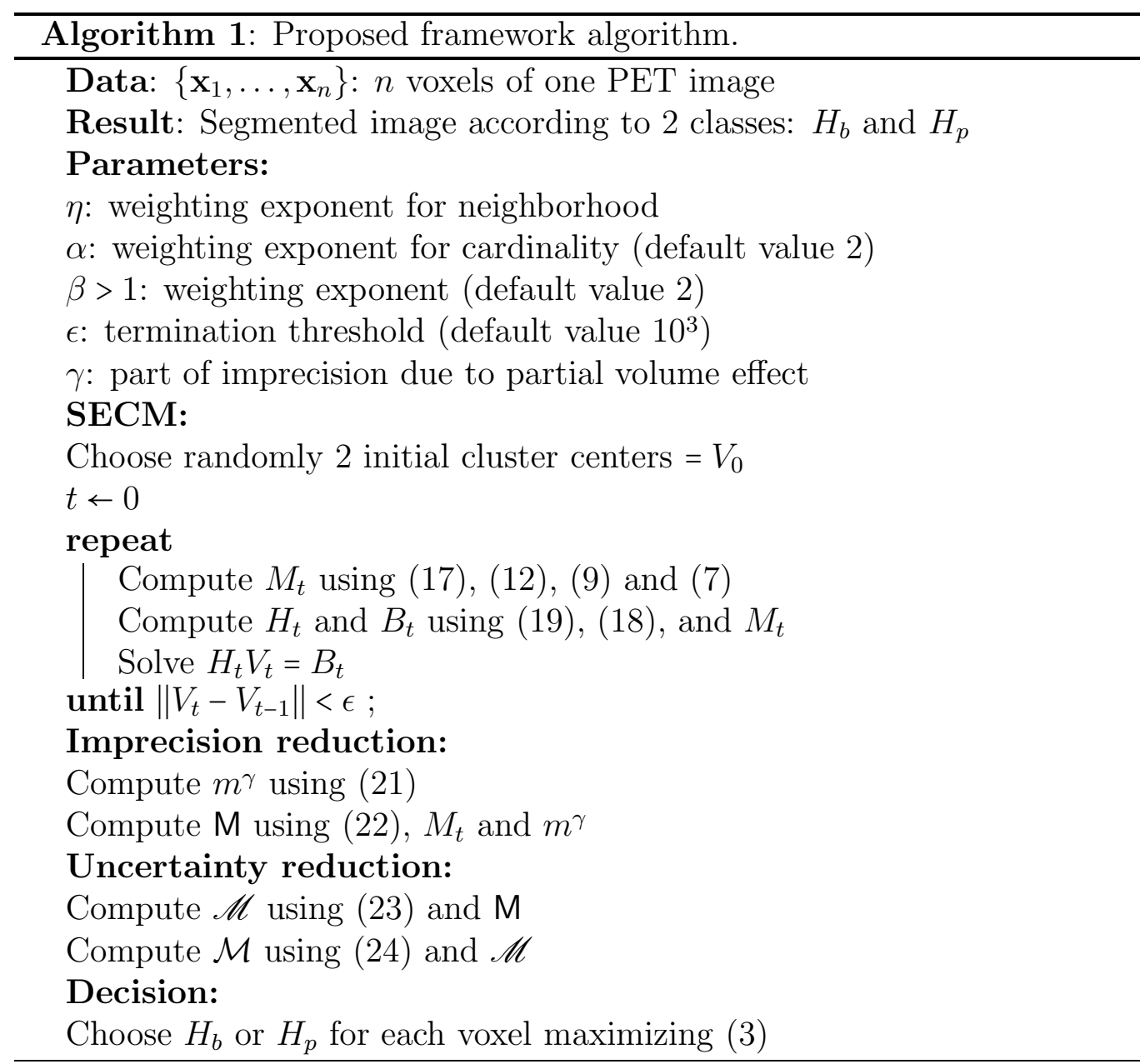

\subsubsection{Fusion for multi-tracer PET images}

As each PET image provides different information on tumor, the fusion of multi-tracer PET images allows us to carry out the dose painting more efficiently.

Frame of discernment. We consider that the FDG PET image is the reference. It means that the positive tissue volume with FLT and FMiso PET images correspond to sub-volumes of the positive tissue volume with FDG PET image. Considering the fact that diagnosis of cell malignancy is clinically done on FDG PET images, and those using FLT and FMizo are supplementary information, FDG PET images have been considered as the reference and FLT and FMizo images as sub-volumes 


\begin{tabular}{c|cc}
\hline Image & $\left\{H_{b}\right\}$ & $\left\{H_{p}\right\}$ \\
\hline FDG & $\{N\}$ & $\{M, P, H, F\}$ \\
FLT & $\{N, M, H\}$ & $\{P, F\}$ \\
FMiso & $\{N, M, P\}$ & $\{H, F\}$
\end{tabular}

Table 1: Hypotheses considered after SECM for multi-modal PET images.

of those defined on FDG PET images. Thus, five classes are distinguished:

- $\{N\}$ corresponds to Normal tissues (i.e., background with FDG).

- $\{M\}$ corresponds to tissues presenting an important glucose Metabolism (i.e., positive tissue with FDG only).

- $\{P\}$ corresponds to tissues with an important cell Proliferation (i.e., positive tissue with FDG and FLT).

- $\{H\}$ corresponds to Hypoxic tissues (i.e., positive tissue with FDG and FMiso).

- $\{F\}$ for $F$ ull uptake corresponds to tissues presenting the three phenomena (i.e., positive tissue with FDG, FLT and FMiso).

The frame of discernment for the three PET images is now: $\Omega=\{N, M, P, H, F\}$. For each voxel of the three modalities, belief masses are spread over 32 hypotheses: $\{\varnothing,\{N\},\{M\},\{P\}, \ldots,\{N, M\}, \ldots, \Omega\}$.

SECM and imperfection reduction. After SECM and imperfection reduction for each PET image, the focal sets are $\left\{H_{b}\right\},\left\{H_{p}\right\}$, and $\left\{H_{b}, H_{p}\right\}$. Because of the frame of discernment used for multi-tracer PET image fusion, and according to the medical interpretation of PET images, the useful hypotheses forming the frame of discernment are given in Table 1 . It is assumed that a low uptake in FDG corresponds to a normal tissue $\{N\}$, while a high uptake in FDG corresponds to a tumoral tissue that can possibly corresponds to a high or low uptake in FLT or FMiso. Moreover, a low uptake in FLT or in FMiso does not mean that the tissue is normal. 
Fusion of multi-tracer PET images for parametric image construction. For the fusion of multi-tracer PET images, we propose fusing each voxel of the three PET images using the conjunctive combination rule (Eq. 5). This combination allows us to discriminate between hypotheses $\{M\},\{P\},\{H\}$ and $\{F\}$ which are very helpful for dose painting.

Decision. Finally, a labeling process is carried out by maximizing the plausibility function according to hypotheses $\{N\},\{M\},\{P\},\{H\}$ and $\{F\}$. This process yields a parametric image presenting the multiple BTVs.

\subsection{Datasets}

\subsubsection{PET phantom images}

Our method was evaluated on two physical PET phantoms. The first one presents a level of noise similar to that encountered on the FDG and FLT PET images. The second presents a level of noise similar to that encountered on the FMiso PET image.

The FDG and FLT phantom contains nine spheres whose volume varies between $0.43 \mathrm{~mL}$ and $97.3 \mathrm{~mL}$. Moreover, five contrasts varying from 2 to 8 are considered. Because the smaller sphere $(0.43 \mathrm{~mL})$ was not visible on the two lower contrasts, and the $0.99 \mathrm{~mL}$ sphere was not visible on the lower contrast, these three spheres have not been considered.

The FMiso phantom contains six spheres whose volumes vary between $0.54 \mathrm{~mL}$ and $26.2 \mathrm{~mL}$. Three contrasts varying from 2.7 to 3.8 were considered. For comparison, the background activity concentration with the FMiso phantom was 17 times lower than that of the FDG and FLT phantom. As a result, the noise was more important in this second phantom. The smallest spheres $(0.54$ and $1.15 \mathrm{~mL}$ ) have not been considered because they are difficult to visualize. For the same reason, the $2.63 \mathrm{~mL}$ sphere has not been considered with the smallest contrast (2.7).

Acquisition parameters for these two phantoms are given in Appendix B.

\subsubsection{Multi-tracer PET images of patients}

PET images of patients using FDG, FLT and FMiso tracers were also used for the evaluation of our method. The images were obtained from three patients suffering from lung cancer in the RTEP4 clinical trial (Vera et al., 2011). As tumors were paramediastinal, no respiratory motion were taken into account. 
Registration of images was made as follow. First, a registration of CT images, acquired for each PET/CT exam, was performed using mutual information (Viola and Wells, 1997) with FDG PET/CT image as reference. Then, registration parameters were used for PET image registration. A transverse slice is given in Figure 4 for three radio-tracers (patient 1). Each image was scaled to its own maximum Standard Uptake Value (SUV).

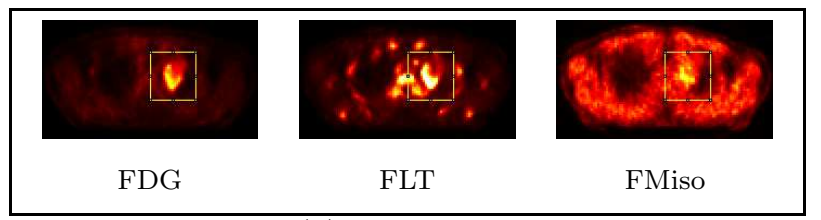

(a) patient \#1

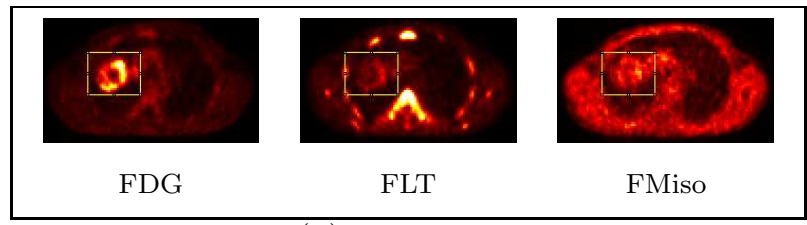

(b) patient \#2

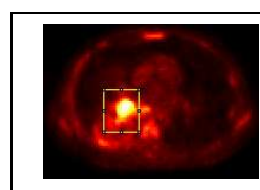

FDG

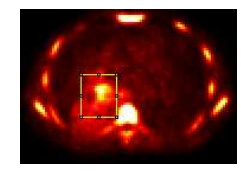

FLT

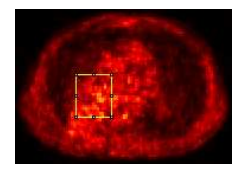

FMiso

(c) patient \#3

Figure 4: FDG, FLT and FMiso PET images for three patients.

\subsection{Approaches used for comparison}

We compared our method on PET phantom images with two other methods recently proposed. The first method is the Adaptive Thresholding (AT) proposed in (Vauclin et al., 2009) for tumor segmentation in FDG PET imaging and inspired from (Daisne et al., 2003). The second method is the Fuzzy Locally Adaptive Bayesian (FLAB) method introduced in (Hatt et al., 2009), with the following parameters: two classes were used, the size of the neighborhood was $3 \times 3 \times 3$ and Gaussian distributions were assumed. Both methods have been proposed for the segmentation of PET images. 


\subsection{Region of interest definition}

As our aim is not to detect tumors, but to estimate their volume, we assume that the lesions have been previously identified by the clinician and that a box encompassing them has manually or automatically been placed. Note that small changes in the placement of the box or its size have no significant impact on the estimation of tumor volume using our method.

\section{Results}

\subsection{Parameter setting}

As mentioned in the previous section, four parameters have to be initialized before applying our method. The first two parameters are $\alpha$ and $\beta$, which control, respectively the degree of penalization of the subsets of high cardinality and the fuzziness degree. Because only three focal sets are used for segmenting PET images $\left(\left\{H_{b}\right\},\left\{H_{p}\right\}\right.$ and $\left.\left\{H_{b}, H_{p}\right\}\right), \alpha$ acts as a weight for hypothesis $\left\{H_{b}, H_{p}\right\}$, and $\beta$ controls the flattening of the fuzzy transition represented by $\left\{H_{b}, H_{p}\right\}$ hypothesis. Because we do not wish to favor $\left\{H_{b}, H_{p}\right\}, \alpha$ has been set to its default value of 2. Coefficient $\beta$ has been set to 6 . This value was chosen empirically using phantom data. Compared to the default value 2, changing the value to 6 has no influence in segmenting positive tissues of large volume, but has an influence on the segmentation of small volumes, which are more subject to partial volume effect. The third parameter $\eta$ controls the influence of the neighborhood. It is related to the spatial resolution of images. Because $F W H M$ is a measure of the spatial resolution of our PET images, and equals $7 \mathrm{~mm}$, this measure was used for initializing $\eta$, as presented in Section 2.3. The last parameter $\gamma$ is used for reducing imprecision in PET images. As explained in Section 2.4.5, its value is determined using PET phantom images.

\subsection{On PET phantom images}

The results of the sphere volume estimation for each method is presented in Figures 5 and 6. Figure 5 shows the results for the FDG and FLT phantom, and Figure 6 for the FMiso phantom. Blue, green and red colors are used, respectively, for SECM, FLAB and AT methods. From left to right, the measured volumes are given from the smallest to the largest sphere. As 


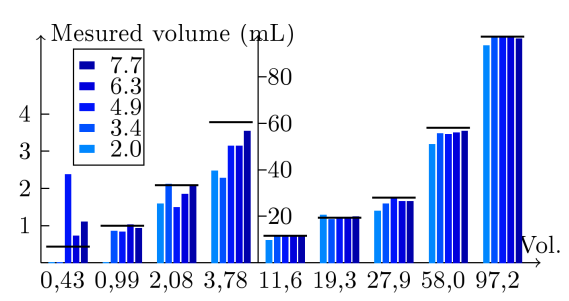

(a) SECM

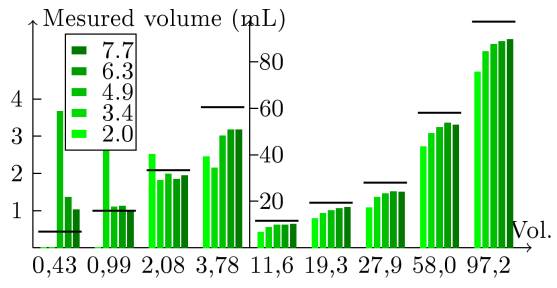

(b) FLAB (Hatt et al., 2009)

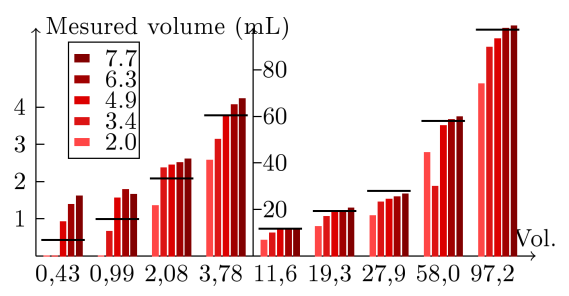

(c) AT (Vauclin et al., 2009)

Figure 5: Histograms of measured volumes according to the actual sphere volumes for SECM (a), FLAB (b) and AT (c) methods. The actual volumes are also identified by horizontal lines. The figure is better viewed in color.

an indication, the true volumes are represented by a horizontal line for each sphere. The closer the color bar and the line, the better the volume estimation. The measured volumes of each sphere are also given for each contrast. The lighter bar corresponds to the lowest contrasts, and the darker bar corresponds to the highest contrasts.

As we can see on the FDG and FLT phantom PET images (Figure 5), SECM provides the best estimation of sphere volumes. For the largest spheres $(>11 \mathrm{~mL}$ ), the mean absolute error is about $1.6 \pm 1.8 \mathrm{~mL}$ for the SECM method, while it is about $6.4 \pm 4.7 \mathrm{~mL}$ and $5 \pm 7.1 \mathrm{~mL}$ for FLAB and AT, respectively. For the smallest spheres $(<4 \mathrm{~mL})$, SECM and FLAB yield comparable results. For these two methods, the errors are due to the high partial volume effect that makes the class barycenter estimation difficult. About AT, for small and for large spheres, the measured volumes increase with respect to the contrast. This dependence on contrast variation shows the poor robustness of the method, although its calibration step uses contrast information. The good performance of our method is due to our strategy of first modeling the imperfections and then reducing them according to the volume of positive tissue and its contrast with the background.

On FMiso phantom PET images of low signal to noise ratio (Figure 6), 


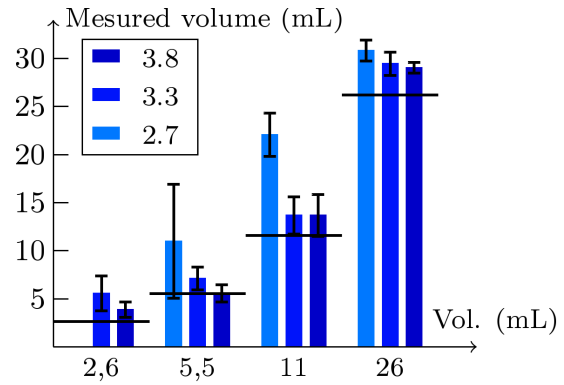

(a) SECM

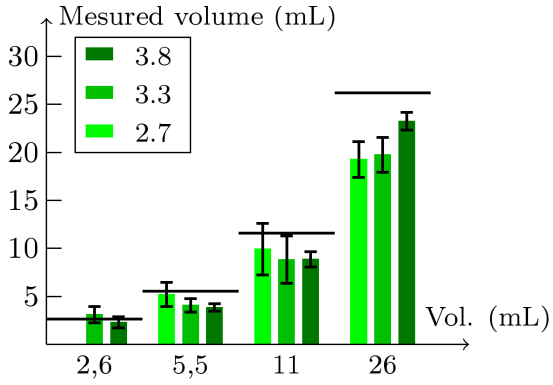

(b) FLAB (Hatt et al., 2009)

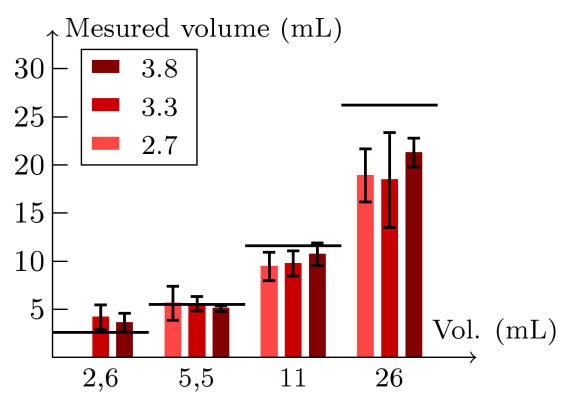

(c) AT (Vauclin et al., 2009)

Figure 6: Histograms of mean measured volumes and their standard deviation according to the actual sphere volumes for SECM (a), FLAB (b) and AT (c) methods. The actual volumes are also identified by horizontal lines. The figure is better viewed in color.

we can see the limit of our SECM method. When a very important noise is present, and when the contrast is very low (about 2.7), SECM gives the worst results. This is due to the very important noise for 2.7 contrast images that induces the presence of regions around the spheres presenting high uptakes. These regions are considered by our method as regions that could potentially correspond to heterogeneity of the positive tissue to segment. That is why an over-estimation of volumes is observed with our method for the lower SNR. However considering 3.3 and 3.8 contrasts only, SECM is the only one method that is robust to sphere size variations. Indeed, the maximum of mean volume error estimations is $+3.2 \mathrm{~mL}$ for SECM (obtained for the smallest sphere), is $-6.5 \mathrm{~mL}$ for FLAB (obtained for the largest sphere) and $-7.7 \mathrm{~mL}$ for AT (obtained for the largest sphere). 


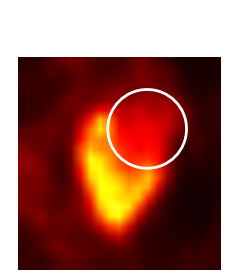

(a) FDG

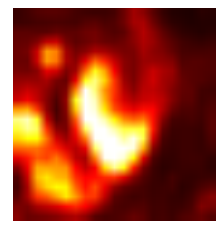

(c) FLT

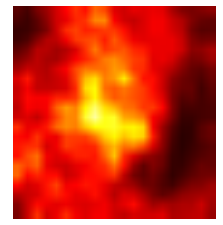

(e) FMiso

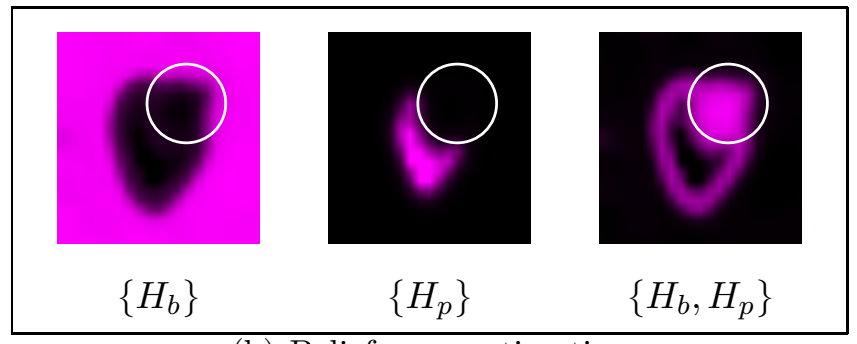

(b) Belief mass estimation

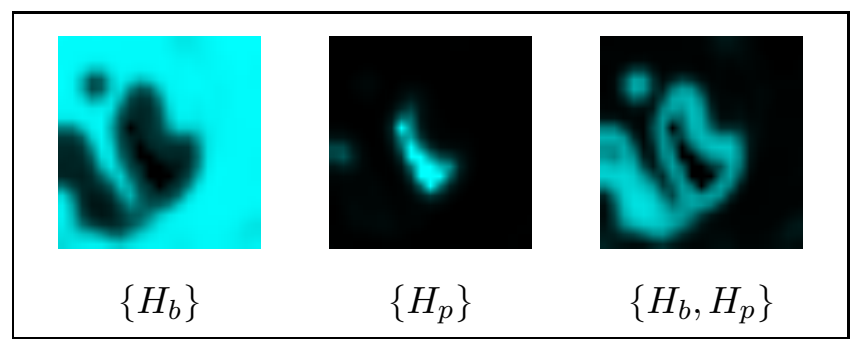

(d) Belief mass estimation

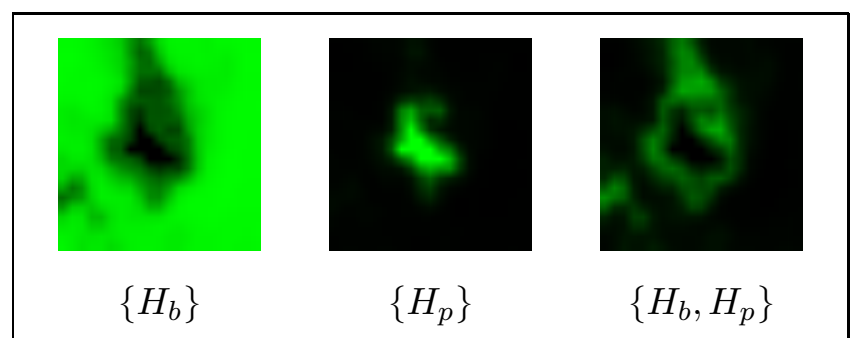

(f) Belief mass estimation

Figure 7: Representation of the three transverse slices of the three radio-tracers and the corresponding belief mass estimations according to hypotheses $\left\{H_{b}\right\},\left\{H_{p}\right\}$ and $\left\{H_{b}, H_{p}\right\}$ using SECM

\subsection{On patient data}

The results of the belief mass estimation using SECM (see section 2.4.1) are given in Figure 7 for patient 1 according to the three-tracer PET images. The belief mass estimation is represented for each tracer PET image by a map according to the three hypotheses, namely $\left\{H_{b}\right\},\left\{H_{p}\right\}$ and $\left\{H_{b}, H_{p}\right\}$. On each map, the lighter (respectively, the darker) the intensity of the color is, the higher (respectively the lower) the mass of the corresponding hypothesis is. A high mass for hypotheses $\left\{H_{b}\right\}$ or $\left\{H_{p}\right\}$ means that the voxel certainly belongs to the corresponding hypothesis. For positive tissues, the high masses are found for voxels for which both themself and their neighborhood present 
a high uptake. They are mainly found in the middle of positive tissues for the three tracers. A voxel having a high mass assigned to $\left\{H_{b}, H_{p}\right\}$ means that it corresponds to an imprecise region. For the three tracers, high masses are in favor of this hypothesis at the transition between the background and positive tissue. It corresponds to voxels that are subject to partial volume effect. Furthermore, as we can see on the FDG belief mass estimation (see Figure $7(\mathrm{~b})$ ), the voxels identified in the white circle have high masses in favor of $\left\{H_{b}, H_{p}\right\}$. It is an imprecise region whose voxels and their neighbors have a similar uptake. It seems to be due to the presence of heterogeneity in the tumor. Thus, hypothesis $\left\{H_{b}, H_{p}\right\}$ models both partial volume effect and heterogeneity regions for which it is difficult to make a decision. Medium masses for a voxel on both $\left\{H_{b}\right\}$ and $\left\{H_{p}\right\}$ means that the voxel is uncertain. This phenomenon is mainly present for the FMiso PET image due to high noise inside the positive tissue region.

The result of the imprecision and uncertainty reduction steps is shown in Figure 8 for patient 1 according to the three-tracer PET images. As we can see for the three tracers, low masses are assigned to hypothesis $\left\{H_{b}, H_{p}\right\}$, showing that the imprecision has well been reduced. Moreover, high masses (close to 1 ) are assigned to $\left\{H_{b}\right\}$ and $\left\{H_{p}\right\}$, showing that the uncertainty has also been reduced. The important point to observe is that the heterogeneous region in the upper right of FDG PET positive tissue is now considered as certainly belonging to the tumor.

The result of the fusion of multi-tracer PET images is given in Figure 9 for patient 1. Figure $9(\mathrm{~d})$ shows the result of the pignistic probability transformation of masses obtained after fusion. The pignistic probability maps are given according to $\{M\},\{P\},\{H\}$ and $\{F\}$ hypotheses. As in the previous figure, the lighter the intensity of the color is, the more certain is the voxel belonging to the corresponding hypothesis. Figure 9(f) corresponds to the segmented regions obtained by taking the maximum plausibility among the five classes. The colors are defined as follows: the black region corresponds to $\{N\}$, the pink region corresponds to $\{M\}$, the blue region corresponds to $\{P\}$, the green region corresponds to $\{H\}$ and the yellow region corresponds to $\{F\}$. Thus, the fusion allows us to obtain the maps of the specific regions to be irradiated for dose painting task. As a high FDG uptake corresponds to tumor cells, FDG PET images are considered as the reference. Tumor cells are not necessarily associated to a high proliferation, nor to hypoxic cells. It is also possible that low-FDG uptake is associated to high-FLT or FMiso uptake. With our fusion 


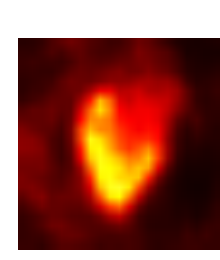

(a) FDG

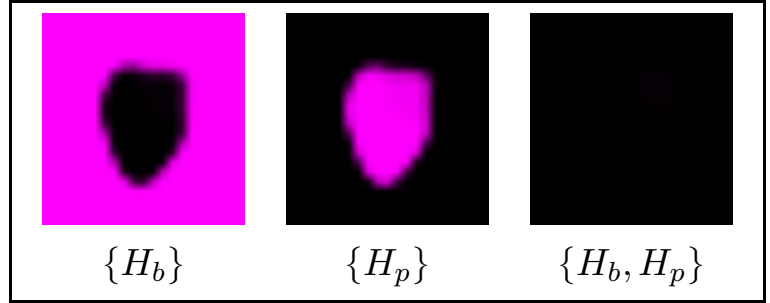

(b) Belief mass estimation

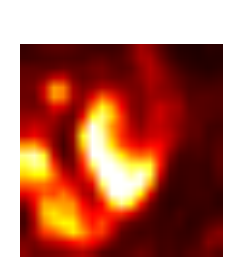

(c) FLT

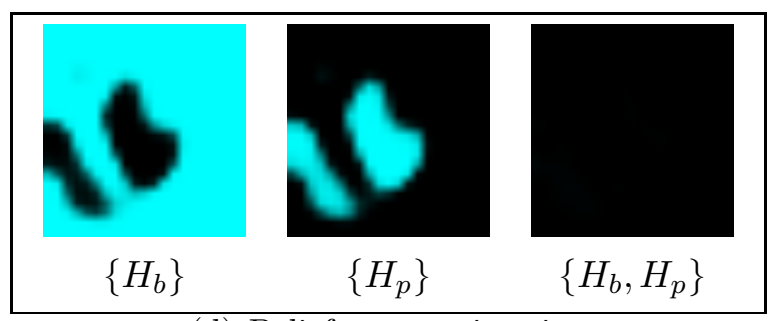

(d) Belief mass estimation

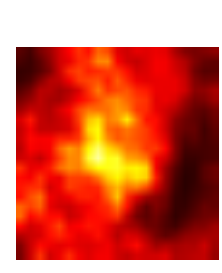

(e) FMiso

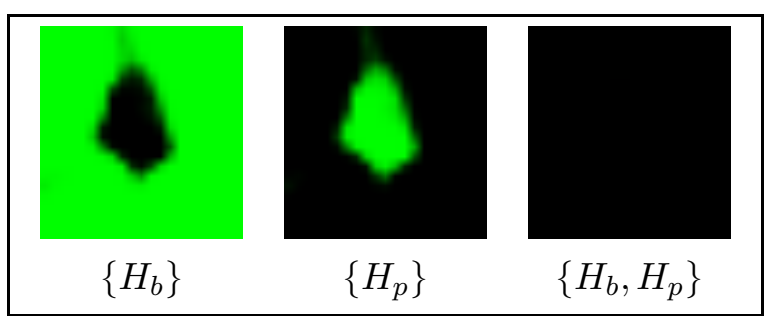

(f) Belief mass estimation

Figure 8: Representation of the three transverse slices of the three radio-tracers and the corresponding belief mass estimations according to $\left\{H_{b}\right\},\left\{H_{p}\right\}$ and $\left\{H_{b}, H_{p}\right\}$ hypotheses after uncertainty and imprecision reduction steps.

model, this kind of information can be seen on a map corresponding to conflict between data. As presented in Figure 9(e), this map (on the empty set) is also highlighted. The conflict corresponds to hypoxic tissues and/or tissues presenting a high cell proliferation that are normal according to glucose metabolism (i.e., positive tissues according to FLT and FMiso and the background with FDG). It shows positive tissues that potentially correspond to healthy tissues. In our case, conflict indicates the presence of bones that have a naturally high cell proliferation. This conflict is interesting information for dose painting because it could correspond to an organ at risk that would need a low radiation dose.

The fusion results for the two other patients are given in Figures 10 


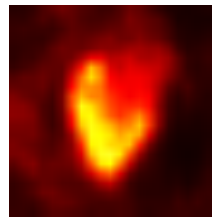

(a) FDG

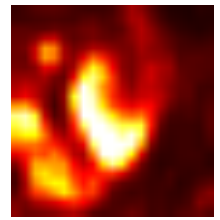

(b) FLT

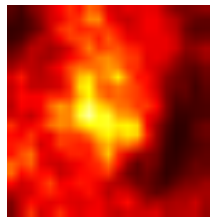

(c) FMiso

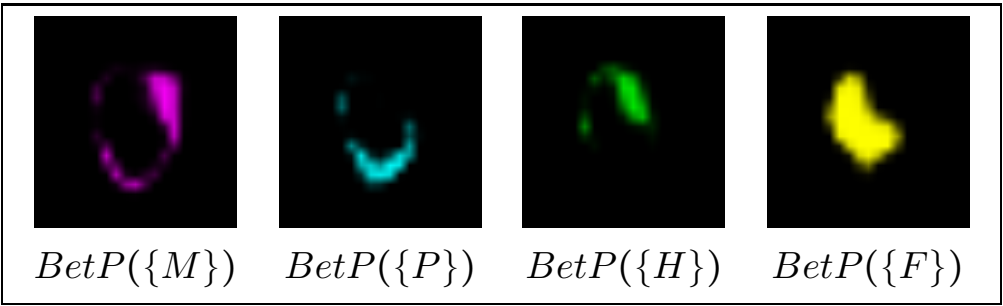

(d) pignistic probabilities

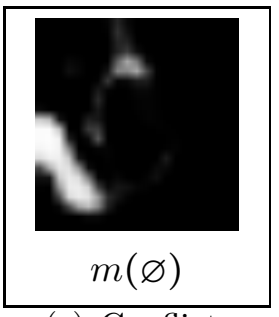

(e) Conflict

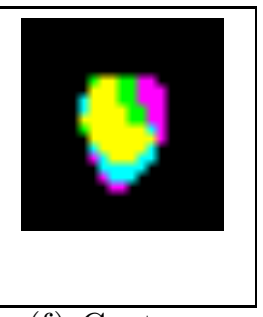

(f) Contours

Figure 9: Images showing results of the method of information fusion on multi-tracer PET images. (a), (b) and (c) are the initial images using FDG, FLT and FMiso tracers. (d) are the parametric images applying first the BBA estimation of each ROI of each image and then fusing these BBAs. The final result showing how dose painting can be achieved is presented in (f); it corresponds to the segmented regions.

and 11. In Figure 10, a necrosis in the tumor region is present and only a small region at the right side of the tumor presents a high cell proliferation associated with a hypoxia. In Figure 11, we observe that the tumor presents a high cell proliferation, but only few voxels correspond to hypoxia. These results show that our method is efficient even if the size of the region to segment is small. They have been visually evaluated by experts.

The use of multiple information conveyed by multi-tracer PET images could have a major impact on the treatment by radiation therapy. The integration of images into a radiation therapy protocol becomes more difficult as the number of images increases. Our results prove that this task can be performed using a fusion 


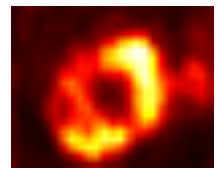

(a) FDG

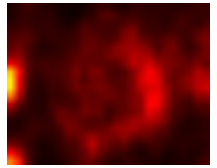

(b) FLT

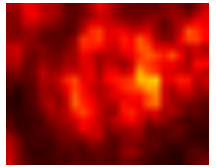

(c) FMiso

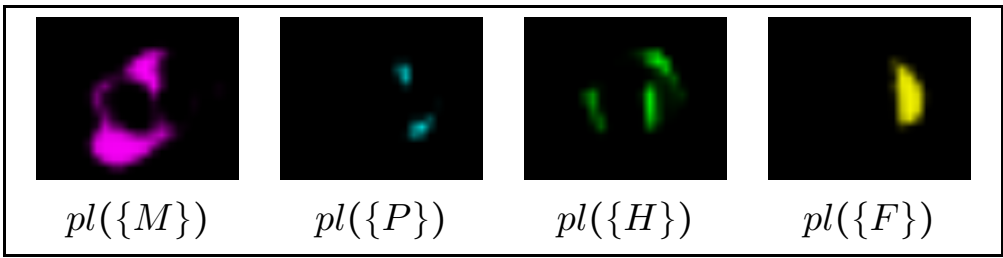

(d) Plausibilities

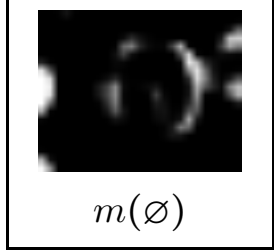

(e) Conflict

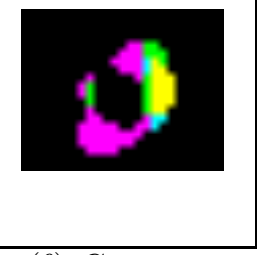

(f) Contours

Figure 10: Images showing results of the method of information fusion for the second patient.

process. Our fusion method may be routinely used in the future.

\section{Conclusion}

In radiotherapy planning, the conventional FDG tracer (glucose metabolism) is broadly used for tumor depiction in PET imaging. However, in many complex situations, new tracers such as FLT and FMISO can provide complementary information: cellular proliferation and hypoxia, respectively. To date, the segmentation is mainly performed on FGD PET images. In this paper we have proposed a framework based on belief function theory to fuse multi-PET tracers. Because the neighborhood information is integrated in the SECM algorithm, our method allows us to model both uncertainty due to noise and imprecision due to partial volume effect and heterogeneity. A process for reducing the uncertainty and the imprecision is proposed, based on a measurement of partial volume effect from the acquisition system. With multi-tracer PET images, a final fusion step is performed to obtain parameter maps that represent different phenomena observed from multi-tracer PET 


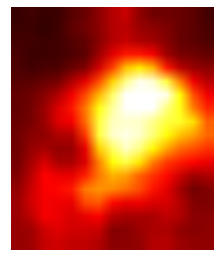

(a) FDG

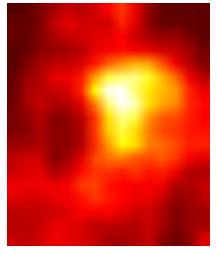

(b) FLT

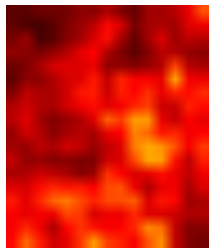

(c) FMiso

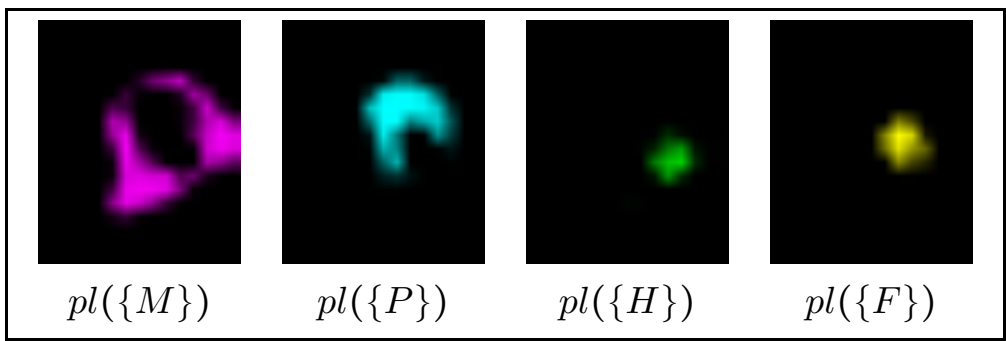

(d) Plausibilities

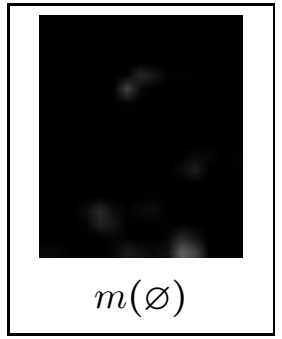

(e) Conflict

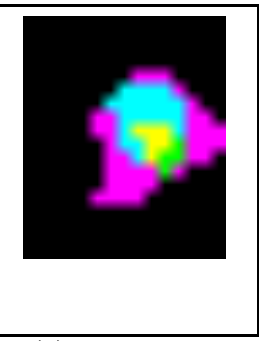

(f) Contours

Figure 11: Images showing results of the method of information fusion for the second patient.

images. These maps allow us to help the radiation oncologist for the treatment planning because it is possible to adapt the dose prescription according to the different sub-volume defining the tumor.

Our method for volume estimation analysis has been validated using two PET phantom images by comparison with a Bayesian (FLAB) and a thresholding (AT) method. On the first phantom, the results have shown that our method gives the best results for the largest spheres $(>11 \mathrm{~mL})$, and comparable results with FLAB for the smallest spheres $(<4 \mathrm{~mL})$. On the second phantom with low SNR, the results has shown that our segmentation method can achieve a better estimation of the sphere volumes when the contrast is higher than 2.7. The poor result with the 2.7 contrast is due to the 
presence of regions around the spheres presenting high uptake due to noise. These regions are considered by our method as regions that could potentially correspond to the positive tissue to segment.

The major interest of our method is that it can be applied either for modeling uncertainty and imprecision, for segmenting BTV by reducing imperfection or for segmenting BTV from multi-tracer PET images by fusing them. The results on patient data show the feasibility of using our method for dose painting. Our method is a valuable help to adapt the dose prescription according to the different sub-volume defining the tumor. This information fusion process from multi-tracer PET images had never been proposed before.

The results were also evaluated using phantom data, which are similar as the SNR found on FDG and FLT, and FMiso PET images. Future work will address the evaluation of our method on heterogeneous simulated phantom data. A dose painting strategy is under validation (Aerts et al., 2010). The impact of our method for treatment planning will be evaluated in our feature work.

\section{Appendix A. SECM optimization}

First, we consider that $V$ is fixed, and we introduce $n$ Lagrange multipliers $\lambda_{i}$ to solve the minimization problem with respect to $M$ :

$$
\mathscr{L}\left(M, \lambda_{1}, \ldots, \lambda_{n}\right)=J_{S E C M}(M, V)-\sum_{i}^{n} \lambda_{i}\left(\sum_{j / A_{j} \neq \varnothing, A_{j} \subseteq \Omega} m_{i j}-1\right)
$$

By differentiating the Lagrangian with respect to the $m_{i j}$ and $\lambda_{i}$ and setting the derivatives to zero, we obtain:

$$
\begin{gathered}
\frac{\partial \mathscr{L}}{\partial m_{i j}}=\beta c_{j}^{\alpha} \cdot m_{i j}^{\beta-1} \sum_{t \in \Phi(i)} b_{t} d_{t j}^{2}-\lambda_{i}=0 \\
\frac{\partial \mathscr{L}}{\partial \lambda_{i}}=\sum_{\left\{j / A_{j} \neq \varnothing, A_{j} \subseteq \Omega\right\}} m_{i j}-1=0
\end{gathered}
$$

We thus have from (A.2):

$$
m_{i j}=\left(\frac{\lambda_{i}}{\beta}\right)^{\frac{1}{\beta-1}}\left(\frac{1}{c_{j}^{\alpha} \sum_{t \in \Phi(i)} b_{t} d_{t j}^{2}}\right)^{\frac{1}{\beta-1}}
$$


Using (A.3) and (A.4):

$$
\left(\frac{\lambda_{i}}{\beta}\right)^{\frac{1}{\beta-1}}=\left(\sum_{j} \frac{1}{c_{j}^{\alpha /(\beta-1)}} \frac{1}{\sum_{t \in \Phi(i)} b_{t}^{1 /(\beta-1)} d_{t j}^{2 /(\beta-1)}}\right)^{-1}
$$

Returning in (A.4), one obtains the necessary condition of optimality for $M$ :

$$
m_{i j}=\frac{c_{j}^{-\alpha / \beta-1}\left(\sum_{t \in \Phi(i)} b_{t}^{1 /(\beta-1)} d_{t j}^{2 /(\beta-1)}\right)^{-1}}{\sum_{A_{k} \neq \varnothing} c_{k}^{-\alpha / \beta-1}\left(\sum_{t \in \Phi(i)} b_{t}^{1 /(\beta-1)} d_{t k}^{2 /(\beta-1)}\right)^{-1}}
$$

Let us now consider that $M$ is fixed. The minimization of $J_{S E C M}$ with respect to $V$ is an unconstrained optimization problem. The partial derivatives of $J_{S E C M}$ with respect to the centers are given by:

$$
\begin{gathered}
\frac{\partial J_{S E C M}}{\partial \mathbf{v}_{l}}=\sum_{i}^{n} \sum_{j} c_{j}^{\alpha} m_{i j}^{\beta} \sum_{t \in \Phi_{i}} b_{t} \frac{\partial d_{i j}^{2}}{\partial \mathbf{v}_{l}} \\
\frac{\partial d_{i j}^{2}}{\partial v_{l}}=2\left(s_{l j}\right)\left(\overline{\mathbf{v}}_{j}-x_{t}\right)\left(\frac{1}{c_{j}}\right) \\
\frac{\partial\left(e^{-\left(\mathbf{v}_{k}-\mathbf{v}_{r}\right)^{2}}\right)}{\partial \mathbf{v}_{l}}=-2\left(\mathbf{v}_{k}-\mathbf{v}_{r}\right) e^{-\left(\mathbf{v}_{k}-\mathbf{v}_{r}\right)^{2}}
\end{gathered}
$$

From (A.7), (A.8) and (A.9), we thus have:

$$
\begin{array}{r}
\frac{\partial J_{S E C M}}{\partial \mathbf{v}_{l}}=2 \sum_{i}^{n} \sum_{A_{j} \neq \varnothing} c_{j}^{\alpha-1} m_{i j}^{\beta} \sum_{t \in \Phi_{i}} b_{t} s_{l j}\left(\overline{\mathbf{v}}_{j}-x_{t}\right) \\
\frac{\partial J_{S E C M}}{\partial \mathbf{v}_{l}}=2 \sum_{i} \sum_{j} c_{j}^{\alpha-1} m_{i, j}^{\beta} \sum_{t \in \Phi_{i}} b_{t} s_{l, j}\left(\frac{1}{c_{j}} \sum_{k} s_{k, j} \mathbf{v}_{k}-x_{t}\right)
\end{array}
$$

Setting these derivatives to zero gives $l$ linear equations in $\mathbf{v}_{k}$ which can be written as:

$$
\sum_{i} \sum_{t \in \Phi_{i}} b_{t} x_{t} \sum_{j} c_{j}^{\alpha-1} m_{i, j}^{\beta} s_{l, j}=\sum_{k} \mathbf{v}_{k} \sum_{i} \sum_{j} c_{j}^{\alpha-2} m_{i, j}^{\beta} s_{l, j} s_{k, j} \sum_{t \in \Phi_{i}} b_{t}
$$

Let $B$ be a matrix of size $(c \times p)$ defined by: 


$$
B_{l q}=\sum_{i} \sum_{t \in \Phi_{i}} b_{t} x_{t q} \sum_{A_{j} \ni \omega_{l}} c_{j}^{\alpha-1} m_{i j}^{\beta}
$$

$l=1, c, q=1, p$, and $H$ a matrix of size $(c \times c)$ given by:

$$
H_{l k}=\sum_{i} \sum_{A_{j} \supseteq\left\{\omega_{k}, \omega_{l}\right\}} c_{j}^{\alpha-2} m_{i j}^{\beta} \sum_{t \in \Phi_{i}} b_{t}
$$

$k, l=1, c$.

$V$ is solution of the following linear system:

$$
H V=B
$$

\section{Appendix B. PET phantom acquisition parameters}

\section{Appendix B.1. FDG and FLT phantom}

Data have been acquired on a Jaszczak phantom (Data Spectrum Corporation $^{\mathrm{TM}}$, Hillsborough, NC, USA). It contains nine spheres whose volume varies between $0.43 \mathrm{~mL}$ and $97.3 \mathrm{~mL}(0.43,0.99,2.08,3.78,11.6,19.3,27.9$, 58.1 and $97.3 \mathrm{~mL}$ ). At the beginning of the acquisition, the activity concentration of the spheres was of $41.6 \mathrm{kBq} / \mathrm{mL}$, whereas the activity concentration of the background was $5.4 \mathrm{kBq} / \mathrm{mL}$. For comparison, similar concentrations are encountered on FDG and FLT PET exams (5.0 MBq/kg). Then, activity has successively been added to the background to make acquisitions with 5 different contrasts $(7.7,6.3,4.9,3.4$ and 2.0).

PET acquisitions and image reconstruction were obtained on a Biograph Sensation 16 Hi-Rez (SIEMENS Medical Solution, Knoxville, TN, USA) following the protocol used in clinical routine. PET emission data were acquired in 3D mode with a $162 \mathrm{~mm}$ axial Field Of View (FOV). After the acquisition, the data were corrected for dead-time, random, scatter and attenuation, and reconstructed with an Attenuation Weighted Ordered Subset Expectation Maximization method (AWOSEM: 4 iterations and 8 subsets) in a $168 \times 168$ matrix (voxel size of $4.06 \times 4.06 \times 2.0 \mathrm{~mm} 3$. A Gaussian post-filtering was applied with a full width at half maximum of $5 \mathrm{~mm}$. The spatial resolution was $6.8 \mathrm{~mm}$ in transverse plane at the center of the FOV. 


\section{Appendix B.2. FMiso phantom}

Data have been acquired on an IEC NEMA phantom (Data Spectrum Corporation $^{\mathrm{TM}}$, Hillsborough, NC, USA). It contains six spheres whose volumes vary between $0.54 \mathrm{~mL}$ and $26.2 \mathrm{~mL}(0.54,1.15,2.63,5.53,11.6$ et 26.2 $\mathrm{mL})$. At the beginning of the acquisition, the activity concentration of the spheres was $1.2 \mathrm{kBq}$, whereas the activity concentration of the background was $0.31 \mathrm{kBq} / \mathrm{mL}$. The objective is to have low concentrations as those encountered on FMiso PET images. For comparison, the background activity concentration is 17 times lower than that of the FDG and FLT phantom. Then, activity has successively been added to the background to make acquisitions with 3 different contrasts (3.8, 3.3 and 2.7).

Except for acquisition time, the same acquisition and reconstruction parameters were used. Data were acquired in List-mode during 21 minutes, and nine bootstrap samples of 7 minutes each were extracted. 7 minutes is also used for FMiso PET image acquisition time. Due to the low activity concentration in the phantom, and thus the low Signal to Noise Ratio (SNR) in the reconstructed images, the use of nine bootstrap samples allows us to better evaluate the methods with respect to noise, by analyzing reproducibility.

\section{References}

Aerts, H.J., Lambin, P., Ruysscher, D.D., 2010. FDG for dose painting: a rational choice. Radiother Oncol 97, 163-164.

Ahmed, M.N., Yamany, S.M., Mohamed, N., Farag, A.A., Moriarty, T., 2002. A modified fuzzy C-means algorithm for bias field estimation and segmentation of MRI data. IEEE Trans Med Imaging 21, 193-199.

Alber, M., Paulsen, F., Eschmann, S.M., Machulla, H.J., 2003. On biologically conformal boost dose optimization. Phys Med Biol 48, N31-35.

Aristophanous, M., Penney, B.C., Martel, M.K., Pelizzari, C.A., 2007. A Gaussian mixture model for definition of lung tumor volumes in positron emission tomography. Med Phys 34, 4223-4235.

Black, Q.C., Grills, I.S., Kestin, L.L., Wong, C.Y., Wong, J.W., Martinez, A.A., Yan, D., 2004. Defining a radiotherapy target with positron emission tomography. Int. J. Radiat. Oncol. Biol. Phys. 60, 1272-1282. 
Capelle-Laize, A.S., Colot, O., Femandez-Maloigne, C., 2004. Evidential segmentation scheme of multi-echo $\mathrm{mr}$ images for the detection of brain tumors using neighborhood information. Information Fusion 5, 203-216.

Chang, J., Wen, B., Kazanzides, P., Zanzonico, P., Finn, R.D., Fichtinger, G., Ling, C.C., 2009. A robotic system for ${ }^{18}$ F-FMISO PET-guided intratumoral pO² measurements. Med Phys 36, 5301-5309.

Chen, S., Zhang, D., 2004. Robust image segmentation using FCM with spatial constraints based on new kernel-induced distance measure. IEEE Trans Syst Man Cybern B Cybern 34, 1907-1916.

Choi, W., Lee, S.W., Park, S.H., Ryu, J.S., Oh, S.J., Im, K.C., Choi, E.K., Kim, J.H., Jung, S.H., Kim, S., Ahn, S.D., 2010. Planning study for available dose of hypoxic tumor volume using fluorine-18-labeled fluoromisonidazole positron emission tomography for treatment of the head and neck cancer. Radiother Oncol 97, 176-182.

Daisne, J.F., Sibomana, M., Bol, A., Cosnard, G., Lonneux, M., Gregoire, V., 2003. Evaluation of a multimodality image (CT, MRI and PET) coregistration procedure on phantom and head and neck cancer patients: accuracy, reproducibility and consistency. Radiother Oncol 69, 237-245.

Dempster, A., 1967. Upper and lower probabilities induced by a multivalued mapping. Annals of Mathematical Statistics 38, 325-339.

Denœux, T., 2008. Conjunctive and disjunctive combination of belief functions induced by nondistinct bodies of evidence. Artif. Intell. 172, 234-264.

Dewalle-Vignion, A.S., Betrouni, N., Lopes, R., Huglo, D., Stute, S., Vermandel, M., 2011. A New Method for Volume Segmentation of PET Images, Based on Possibility Theory. IEEE Trans Med Imaging 30, 409-423.

Duprez, F., De Neve, W., De Gersem, W., Coghe, M., Madani, I., 2011. Adaptive dose painting by numbers for head-and-neck cancer. Int. J. Radiat. Oncol. Biol. Phys. 80, 1045-1055.

Erdi, Y.E., Mawlawi, O., Larson, S.M., Imbriaco, M., Yeung, H., Finn, R., Humm, J.L., 1997. Segmentation of lung lesion volume by adaptive positron emission tomography image thresholding. Cancer 80, 2505-2509. 
Geets, X., Lee, J.A., Bol, A., Lonneux, M., Gregoire, V., 2007. A gradientbased method for segmenting FDG-PET images: methodology and validation. Eur. J. Nucl. Med. Mol. Imaging 34, 1427-1438.

Green, A.J., Francis, R.J., Baig, S., Begent, R.H., 2008. Semiautomatic volume of interest drawing for (18)F-FDG image analysis-method and preliminary results. Eur. J. Nucl. Med. Mol. Imaging 35, 393-406.

Haase, R., Böhme, H.J., Perrin, R., Zöphel, K., Abolmaali, N., 2012. Selfreproduction versus transition rules in ant colonies for medical volume segmentation, in: Proceedings of the 8th international conference on Swarm Intelligence, Springer-Verlag, Berlin, Heidelberg. pp. 316-323.

Haiying, G., Toshiro, K., Xiaolei, H., Xiang, S.Z., Turk, M., 2006. Automatic hot spot detection and segmentation in whole body FDG-PET images, in: IEEE International Conference on Image Processing, Atlanta, GA. pp. $85-88$.

Hall, E.J., 2005. Dose-painting by numbers: a feasible approach? Lancet Oncol. 6, 66 .

Hatt, M., Cheze-Le Rest, C., Aboagye, O., E., Kenny, M., L., Rosso, L., Turkheimer, E., F., Albarghach, M., N., Metges, J.P., Pradier, O., Visvikis, D., 2010. Reproducibility of ${ }^{18} \mathrm{~F}-\mathrm{FDG}$ and 3'-deoxy-3'-18 F-fluorothymidine PET tumor volume measurements. Journal of nuclear medicine : official publication, Society of Nuclear Medicine 51, 1368-76.

Hatt, M., Cheze le Rest, C., Turzo, A., Roux, C., Visvikis, D., 2009. A fuzzy locally adaptive bayesian segmentation approach for volume determination in PET. IEEE Transactions on Medical Imaging 28, 881-893.

Krohn, T., Kaiser, H.J., Gagel, B., Boy, C., Schaefer, W.M., Buell, U., Zimny, M., 2007. [3D volume and SUV analysis of oncological PET studies: a voxel-based image processing tool with NSCLC as example]. Nuklearmedizin 46, 141-148.

Lelandais, B., Gardin, I., Mouchard, L., Vera, P., Ruan, S., 2012. Segmentation of biological target volumes on multi-tracer pet images based on information fusion for achieving dose painting in radiotherapy, in: $15^{\text {th }}$ 
International Conference on Medical Image Computing and Computer Assisted Intervention (MICCAI), Lecture Note in Computer Science, Nice, France. pp. 545-552.

Ling, C.C., Humm, J., Larson, S., Amols, H., Fuks, Z., Leibel, S., Koutcher, J.A., 2000. Towards multidimensional radiotherapy (MD-CRT): Biological imaging and biological conformality. Int. J. Radiat. Oncol. Biol. Phys. 47, $551-560$.

Makni, N., Betrouni, N., Colot, O., 2014. Introducing spatial neighbourhood in Evidential C-Means for segmentation of multi-source images: application to prostate multi-parametric MRI. Information Fusion 19, 61-72.

Masson, M.H., Denœux, T., 2008. Ecm : An evidential version of the fuzzy c-means algorithm. Pattern Recognition 341, 1384-1397.

Nestle, U., Kremp, S., Schaefer-Schuler, A., Sebastian-Welsch, C., Hellwig, D., Rube, C., Kirsch, C.M., 2005. Comparison of different methods for delineation of ${ }^{18} \mathrm{~F}$-FDG PET-positive tissue for target volume definition in radiotherapy of patients with non-Small cell lung cancer. J. Nucl. Med. 46, 1342-1348.

Potesil, V., Huang, X., Zhou, X.S., 2007. Automated tumour delineation using joint pet/ct information, in: Proc. Of SPIE, Medical Imaging: Computer-aided Diagnosis.

Riddell, C., Brigger, P., Carson, R.E., Bacharach, S.L., 1999. The watershed algorithm: a method to segment noisy pet transmission images. IEEE Transactions on Nuclear Science 46, 713-719.

Shafer, G., 1976. A mathematical theory of evidence. Princeton university press.

Smets, P., 1993. Belief functions: The disjunctive rule of combination and the generalized bayesian theorem. International Journal of Approximate Reasoning 9, 1-35.

Smets, P., Kennes, R., 1994. The transferable belief model. Artificial Intelligence 66, 191-234. 
Thorwarth, D., 2012. Radiotherapy treatment planning based on functional PET/CT imaging data. Nuclear Medecine Review 15, 43-47.

Thorwarth, D., Eschmann, S.M., Paulsen, F., Alber, M., 2007. Hypoxia dose painting by numbers: a planning study. Int. J. Radiat. Oncol. Biol. Phys. 68, 291-300.

Tylski, P., Bonniaud, G., Decenciere, E.and Stawiaski, J., Coulot, J., Lefkopoulos, D., Ricard, M., 2006. 18F-FDG PET images segmentation using morphological watershed: a phantom study, in: IEEE Nuclear Science Symposium Conference Record, San Diego. pp. 2063-2067.

Vauclin, S., Doyeux, K., Hapdey, S., Edet-Sanson, A., Vera, P., Gardin, I., 2009. Development of a generic thresholding algorithm for the delineation of ${ }^{18}$ FDG-PET-positive tissue: application to the comparison of three thresholding models. Phys. Med. Biol. 54, 6901-16.

Vera, P., Bohn, P., Edet-Sanson, A., Salles, A., Hapdey, S., Gardin, I., Menard, J.F., Modzelewski, R., Thiberville, L., Dubray, B., 2011. Simultaneous positron emission tomography (PET) assessment of metabolism with ${ }^{18}$ F-fluoro-2-deoxy-d-glucose (FDG), proliferation with ${ }^{18} \mathrm{~F}$-fluorothymidine (FLT), and hypoxia with ${ }^{18}$ fluoro-misonidazole (F-miso) before and during radiotherapy in patients with non-small-cell lung cancer (NSCLC): a pilot study. Radiother Oncol 98, 109-116.

Viola, P., Wells, III, W.M., 1997. Alignment by maximization of mutual information. Int. J. Comput. Vision 24, 137-154.

Yang, W., Zhang, Y., Fu, Z., Yu, J., Sun, X., Mu, D., Han, A., 2010. Imaging of proliferation with ${ }^{18} \mathrm{~F}-\mathrm{FLT} \mathrm{PET} / \mathrm{CT}$ versus ${ }^{18} \mathrm{~F}-\mathrm{FDG} \mathrm{PET} / \mathrm{CT}$ in nonsmall-cell lung cancer. Eur. J. Nucl. Med. Mol. Imaging 37, 1291-1299.

Zaidi, H., Diaz-Gomez, M., Boudraa, A., Slosman, D.O., 2002. Fuzzy clustering-based segmented attenuation correction in whole-body PET imaging. Phys Med Biol 47, 1143-1160.

Zhang, P., Gardin, I., Vannoorenberghe, P., 2007. Information fusion using evidence theory for segmentation of medical images, in: International Colloquium on Information Fusion, pp. 265-272. 